\title{
Cancer-associated myasthenic (Eaton-Lambert) syndrome: distribution of abnormality and effect of
} treatment

\author{
DA INGRAM, GR DAVIS, $\dagger$ MS SCHWARTZ, M TRAUB, AC NEWLAND*, M SWASH \\ From the Departments of Neurology and Haematology, ${ }^{*}$ The London Hospital, and the Department of \\ Electrical and Electronic Engineering, Queen Mary College, $\dagger$ London, UK
}

SUMMARY Treatment with plasma exchange, steroids, immunosuppressant drugs and cytotoxic chemotherapy was effective in two cases of cancer-associated Eaton-Lambert myasthenic syndrome. The clinical improvement noted during treatment was accompanied by improvement in the electrophysiological abnormality; this was much more marked in abductor digiti minimi and trapezius than in extensor digitorum brevis.

The myasthenic syndrome is a disorder of neuromuscular transmission characterised by weakness and muscular atrophy. ${ }^{2}$ Weakness is most marked in the proximal muscles of the legs, and it is generally worse at rest, improving with exercise. Ocular muscles and eyelids are usually spared. Many patients complain of a dry mouth and other autonomic disturbances may occur. ${ }^{1-3}$ These features distinguish the disorder from myasthenia gravis. The diagnosis is confirmed by the characteristic electrophysiological findings. The compound muscle action potential evoked by a single nerve stimulation is of low amplitude, and a decremental response is observed during low-frequency repetitive nerve stimulation. However, stimulation at a faster rate elicits an incremental response, sometimes preceded by an initial decrement. ${ }^{124-6}$

It has been shown that the myasthenic syndrome is a pre-synaptic disorder in which the number of quanta of acetylcholine released by each nerve impulse is reduced. ${ }^{4}$ This results from binding of an IgG autoantibody to nerve terminal determinants. ${ }^{78}$ The suggestion that the disease had an autoimmune basis $^{1}$ led to treatment with plasma exchange and immunosuppressive drugs. ${ }^{7-10}$

In assessing the response to treatment, the distibution of weakness and the electrophysiological

Address for reprint requests: Dr M Swash, The London Hospital, London E1 1BB, UK.

Received 29 November 1983 and in revised form 9 February 1984 Accepted 18 February 1984 abnormality must be carefully considered. Improvement has been reported during treatment with guanidine, ${ }^{5}$ 4-aminopyridine and related compounds ${ }^{1-13}$ and with steroid therapy, ${ }^{14}$ is plasma exchange $^{7-1016}$ and immunosuppressive drugs. ${ }^{891617}$ This improvement has been correlated with an increase in the amplitude of the initial evoked compound muscle action potential. ${ }^{8-1017}$ These studies $^{7-101617}$ have concerned patients with the myasthenic syndrome unrelated to carcinoma, apart from two cases of carcinoma-associated myasthenic syndrome, ${ }^{78}$ and one patient with multiple myeloma. ${ }^{18}$

In this paper we describe the response to treament with plasma exchange and cytotoxic chemotherapy, and the distribution of the clinical and electrophysiological abnormality, in two patients with carcinoma-associated myasthenic syndrome.

\section{Patients}

\section{Case 1}

A 53-year-old man presented to another hospital with a 10 day history of progressive proximal weakness. He was unable to lift his arms to shave adequately or to rise unaided from a squatting position but hand grip remained relatively unaffected. He gradually deteriorated during the following two months. Polymyositis was suspected and treatment was begun with oral prednisolone $60 \mathrm{mg}$ on alternate days. He noticed slight improvement in strength on steroid treatment days but there was no overall improvement and he remained severely disabled. One month after beginning this treatment, and four months after the onset of muscular weakness, he was transferred to The London Hospital. At that time it was noticed that he 
complained of a persistently dry mouth. On examination there was proximal weakness more marked in hips than shoulders. There was mild symmetrical wasting of forearm muscles, deltoids, triceps, quadriceps, hamstring and gastrocnemius muscles. He was strikingly weak on awakening in the morning, improved during the day's activities, but became progressively weaker during the latter half of the day. A transient improvement in strength was noted during a short voluntary contraction in all limb muscle groups. However, during sustained exercise there was marked fatiguability, and he required support when walking more than a few metres. The deep tendon reflexes could only be elicited during the few seconds following a maximal voluntary contraction of the same muscle group. There was no diplopia or ptosis and speech and swallowing were normal. Investigations Serum electrolytes, thyroid function, immunoglobulin electropheresis, complement (C3 and C4) levels and DNA binding were normal. Thyroid and gastric parietal antibodies were not detected. Antinuclear factor estimations showed a diffuse increase. The blood creatine kinase (CK) was $47 \mathrm{IU} / 1$ (normal less than $130 \mathrm{IU} / \mathrm{l}$ ). Tissue typing revealed $A 1,2 / B 8, W 62 / C W 7, W 3 / B W-/ 6$, DR3. Chest radiographs showed slight hilar enlargement and tomography revealed a small mass. Following bronchoscopy, histological confirmation of an oat-cell bronchial carcinoma was obtained. No metastases were found during whole-body isotope scanning. A quadriceps muscle biopsy showed Type 2B fibre atrophy with scattered pointed atrophic Type 1 fibres. Two fibres in the biopsy were necrotic but there were no inflammatory cell exudates. EMG investigations were carried out on several occasions (see below).

Clinical course Treatment with alternate day prednisolone was continued and plasma exchange was begun. Improvement was evident after the third plasma exchange, six days after beginning this treatment. Walking improved more slowly than arm strength, and eight weeks after beginning treatment his strength was virtually normal. Initially he could walk $\mathbf{4 0}$ metres in 59 seconds. After three plasma exchanges (day 14) he could walk this distance in 45 seconds and after 8 exchanges (day 39 ) he could walk it in 35 seconds. On day 56 he also completed the distance in 35 seconds. Plasma exchanges (approximately three litres) were performed on days $9,10,12,16,17,22$, and 25 and chemotherapy, comprising intravenous cyclophosphamide and vincristine, and oral VP16 (100 mg) was given on days $18,39,60$, and 81 . The prednisolone dosage was gradually reduced from 60 to $30 \mathrm{mg}$ on alternate days but at about day 150-160 he noticed a gradual return of weakness and fatiguability. Strength rapidly improved when prednisolone dosage was increased to $50 \mathrm{mg}$ on alternate days.
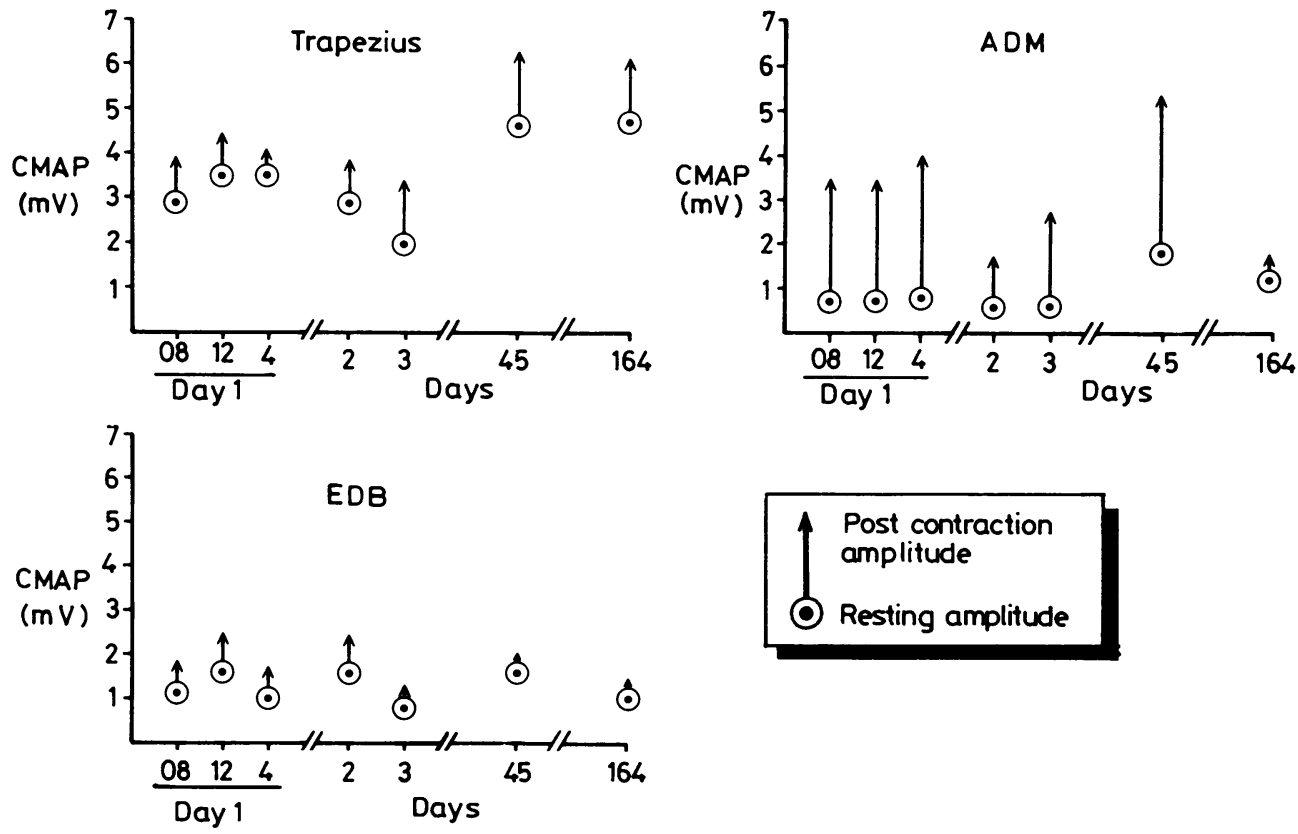

Fig 1 Case 1. Initial compound muscle action potential amplitude after a period of rest and after 30 seconds maximal voluntary contraction in trapezius, abductor digiti minimi and extensor digitorum brevis muscles. Increase in amplitude after voluntary contraction is most evident in abductor digiti minimi. In trapezius and abductor digiti minimi there is a gradual improvement in initial compound muscle action potential amplitude during the course of treatment (normal values given in table 1) 
Case 2

A 58-year-old man, who smoked a hundred cigarettes per day, presented seven months before the development of muscular weakness with a six week history of increasing dyspnoea on exertion, a hoarse voice and prominent neck swelling. He was found to have a mass in the left hilar region with a widened mediastinum. A diagnosis of superior vena cava obstruction due to carcinoma of the bronchus was made. He was treated with radiotherapy to the mediastinum; symptoms of superior vena cava obstruction improved but he was left with a paralysed vocal cord. Muscular weakness developed during the three months before admission, particularly affecting the legs and neck, and he complained that his mouth was dry. On examination there was marked proximal weakness, more marked in the legs, especially the right, with weakness of the trunk and neck. The legs were weaker than the arms and he could walk only a few metres unaided. Distal strength was relatively preserved. There was bilateral ptosis but ocular movements were normal. Swallowing was difficult. The tendon reflexes were absent.

Investigations No abnormality was found on routine haematological and biochemical investigations. The $\mathrm{CK}$ was 53 IU/l. Tissue typing revealed A1, A11/B8, B18/ CW7, $-1 \mathrm{BW} / 6$. A quadriceps muscle biopsy showed disseminated neurogenic atrophy with several zones of Type 1 and Type 2 fibre grouping. In addition, there was a sparse interstitial infiltration with small lymphocytes. EMG studies were carried out on three occasions before and during treatment.

Clinical course Plasma exchange was begun on the 5th hospital day and walking was noticeably improved four days later, after three exchanges. Improvement continued and three weeks later, after six exchanges, strength was normal in the arms but there was only slight improvement in the legs. Cytotoxic chemotherapy was begun with cyclophosphamide, vincristine and VP 16. There was some further improvement in muscular strength during the next four weeks but he then developed pneumonia and died.

\section{Electrophysiological methods}

Routine concentric needle muscle sampling, single fibre EMG examinations, and motor and sensory nerve conduction studies were performed using standard methods with limbs adequately warmed. On days $1,2,3,22,45$ and 164, serial surface-recorded, evoked compound muscle action potentials were obtained in response to repetitive $2 \mathrm{~Hz}$ and $20 \mathrm{~Hz}$ supramaximal stimulation of nerves supplying trapezius $^{19}$ abductor digiti minimi (ADM) and extensor digitorum brevis (EDB) muscles. ${ }^{20} 21$ Care was taken both to ensure adequate rest between trials and to make accurate replication of electrode sites. Recordings were made at rest and following 30 seconds of maximal voluntary (iosmetric) contraction of the appropriate muscle (fig 1). Computer analysis of the compound muscle action potentials $^{22}$ was used to determine amplitude and area measurements, for the $1 \mathrm{st}, 5 \mathrm{th}, 10$ th and 20 th successive potentials in each stimulus train.

Sequential observations were carried out in both patients during treatment (fig 1 ). In Case 1 steroid therapy was discontinued for 48 hours before the investigations. On day 1 recordings were made at 0800 hours (before rising), at 1200 hours after a sedentary morning, and at 1600 hours after exercising to tolerance during the afternoon. Further examinations (days 2, 3, 22, 45 and 164) were performed at 1600 hours without preceding exercise. Day 2 was a steroid treatment day (prednisolone $60 \mathrm{mg}$ at 0800 hours) and the next day (day 3) was a steroid free day. Case 2 was studied on three occasions during the course of diagnosis and treatment. The same muscles were studied as in Case 1.

\section{Results of electrophysiological studies}

Motor and sensory nerve conduction velocities in the median, ulnar and peroneal nerves were within normal limits in both patients. Concentric needle EMG sampling of biceps, quadriceps and tibialis anterior muscles showed low amplitude motor units which tended to increase in amplitude during continous activity. The interference pattern was full. In Case 1 single fibre EMG of the left extensor digitorum communis muscle revealed a number of potential pairs with increased jitter and blocking but a normal fibre density. At higher innervation rates there was less impulse blocking. Evoked compound muscle action potentials to supramaximal stimulation in the three muscles tested in each subject were of reduced amplitude and failed to attain normal values despite the prominent facilitation asssociated with voluntary contraction for 30 seconds (table), or during $20 \mathrm{~Hz}$ electrical stimulation for one second. The reduction in initial amplitude was more marked in abductor digiti minimi and extensor digitorum brevis than in trapezius (table) reduction in initial amplitude.

Table Compound muscle action potential amplitudes before treatment

\begin{tabular}{|c|c|c|c|c|c|c|}
\hline & \multicolumn{3}{|c|}{$\begin{array}{l}\text { Initial compound muscle action potential } \\
\text { amplitude } \mathrm{mV}\end{array}$} & \multicolumn{3}{|c|}{$\begin{array}{l}\text { Compound muscle action potential amplitude after } 30 \mathrm{~s} \\
\text { contraction } \mathrm{mV}(\% \text { change) }\end{array}$} \\
\hline & Trapezius & $A D M$ & $\overline{E D B}$ & Trapezius & $A D M$ & $E D B$ \\
\hline $\begin{array}{l}\text { Case } 1 \\
\text { Case } 2 \\
\text { Normal values }\end{array}$ & $\begin{array}{r}2.9 \\
0.4 \\
>4.0\end{array}$ & $\begin{array}{r}0.6 \\
0.4 \\
>6.0\end{array}$ & $\begin{array}{r}1 \cdot 1 \\
0.3 \\
>2.5\end{array}$ & $\begin{array}{l}3 \cdot 8(35) \\
1.0(250)\end{array}$ & $\begin{array}{l}3.4(467) \\
1 \cdot 3(325)\end{array}$ & $\begin{array}{l}1.8(55) \\
0.3(0)\end{array}$ \\
\hline
\end{tabular}

$\mathrm{ADM}=$ Abductor digiti minimi

$\mathrm{EDB}=$ Extensor digitorum brevis. 
SERIAL ELECTROPHYSIOLOGICAL STUDIES IN CASE 1

Initial compound muscle action potential amplitude On day 1 , when the patient had not received steroids for 48 hours, the initial compound muscle action potential amplitudes were lowest in abductor digiti minimi (10\% of normal), and extensor digitorum brevis ( $44 \%$ of normal), but less abnormal in trapezius ( $73 \%$ of normal). There was a slight increase in the initial compound muscle action potential amplitude in the noon and afternoon studies in the trapezius but little or no diurnal variation occurred in extensor digitorum brevis and abductor digiti minimi (fig 1). On day 2, studies were carried out in the afternoon after an early morning dose of prednisolone; there was no change in the initial compound muscle action potential amplitude compared with the recordings of the previous day.

On day 45, 3 weeks after completion of a course of eight plasma exchanges, and after two cytotoxic treatments, the initial compound muscle action potential amplitudes were increased in two muscles. In the trapezius the amplitude was normal; in the abductor digiti minimi the amplitude was twice the pre-treatment value but there was no change in the extensor digitorum brevis. These changes in initial compound muscle action potential amplitude broadly correlated with the clinical observations of a greater increase in proximal than in distal muscles during the period of treatment. The slight relapse at day 150-160 was accompanied by a reduction in initial compound muscle action potential amplitude in abductor digiti minimi but the trapezius remained normal.

Effect of voluntary activation In all three muscles there was an increase in compound muscle action potential amplitude following voluntary contraction of the tested muscle (fig 1). This was most marked in abductor digiti minimi, in which the compound muscle action potential increased by more than $450 \%$ (table), compared with $55 \%$ change in extensor digitorum brevis and $35 \%$ in trapezius. On day 45 , following plasma exchange and immunosuppressive therapy the post-contraction compound muscle action potential amplitude was normal in trapezius $(6 \mathrm{mV})$ and nearly normal in abductor digiti minimi $(5.2 \mathrm{mV})$ but the response in extensor digitorum brevis remained smaller than normal $(2.0 \mathrm{mV})$.

Effects of repetitive nerve stimulation Repetitive stimulation at $2 \mathrm{~Hz}$ (fig 2) elicited an initial decrement in all the recordings. Stimulation at $20 \mathrm{~Hz}$ in the trapezius and abductor digiti minimi muscles (fig 2) evoked an initial decrement followed by an incremental response, but in the extensor digitorum brevis the response was always incremental after the first stimulus. Although clinical diurnal changes were noted during day 1 , and in relation to steroid treatment (day 2), there was no change in the responses to repetitive nerve stimulation at $2 \mathrm{~Hz}$ and $20 \mathrm{~Hz}$. The incremental response to $20 \mathrm{~Hz}$ repetitive stimulation was usually within normal limits $(40 \%)^{23}$ in trapezius and abductor digiti minimi but was increased in the extensor digitorum
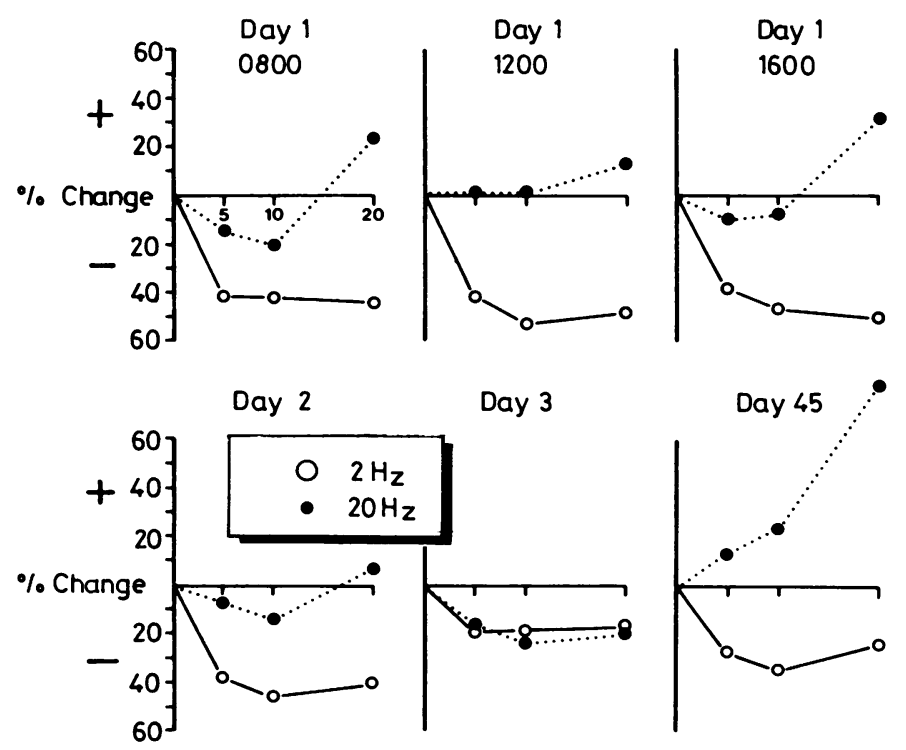

Fig 2 Case 1. Effect of 20 repetitive supramaximal stimulations of the ulnar nerve at $2 \mathrm{~Hz}$ and $20 \mathrm{~Hz}$ on compound muscle action potential in abductor digiti minimi. There was no diurnal variation during day 1 (see text) but a markedly increased and more rapid incremental response on day 45 , after treatment. (area measurements) 

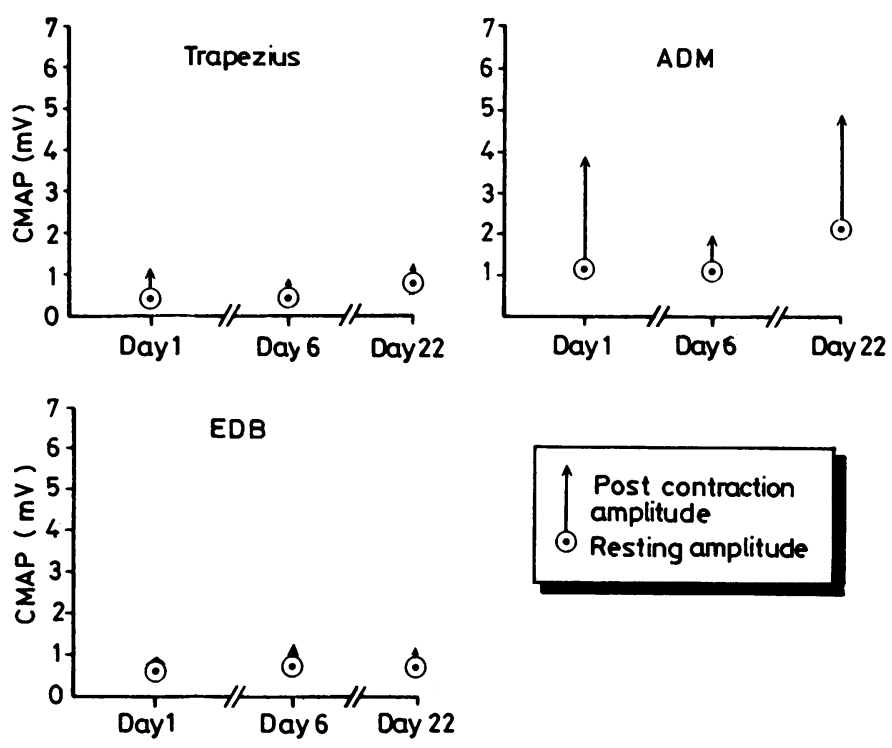

brevis studies. The compound muscle action potential amplitude was reduced in recordings in trapezius and extensor digitorum brevis on day 3 , a day on which steroids were withheld. On day 45 after eight plasma exchanges during the previous 36 days, and immunosuppressive treatment, the largest incremental response was recorded.

\section{SERIAL ELECTROPHYSIOLOGICAL STUDIES IN CASE 2}

Initial compound muscle action potential amplitude The initial responses on day 1 were very small ( $1 \mathrm{mV}$ or less) in all three muscles. By the 22nd day, after completing six plasma exchanges (begun on the fifth day) and one cytotoxic chemotherapy treatment, the initial compound muscle action potential response was more than doubled in abductor digiti minimi, slightly increased in trapezius, but unchanged in extensor digitorum brevis (fig 3 ). During this time the patient's strength and exercise tolerance were improving.

Effects of contraction In the extensor digitorum brevis and trapezius there was only minimal increase in amplitude of the compound muscle action potential following activation, before and after treatment. In abductor digiti minimi there was a marked facilitatory response to activation, which reached a normal amplitude on day 22 , following treatment (fig 3).

\section{Discussion}

Newsom-Davis and others ${ }^{8}$ have suggested that the carcinoma-associated and non-carcinoma- associated forms of the myasthenic syndrome are autoimmune diseases with different triggering factors. In HLA studies in five patients with the noncarcinomatous form they found that all five cases had the DR3 antigen and four showed the HLA-B8 antigen. ${ }^{8}$ Both our patients with myasthenic syndrome associated with bronchogenic carcinoma had HLA-B8 antigens and one (Case 1) also had the DR3 antigen. There is an increased incidence of organ-specific antibodies, particularly of thyroid and parietal cell antibodies in non-carcinoma associated myasthenic syndrome, but not in the carcinomaassociated form of the disease. ${ }^{24}$ Myasthenic syndrome occurs in only a small proportion of patients with oat cell carcinoma of the bronchus. Although Lambert $e t \mathrm{al}^{2}$ estimated an incidence of about $6 \%$ in patients with oat cell carcinoma surveys of unselected patients with oat cell carcinoma suggest that the incidence is much lower. ${ }^{25} 26$ It thus seems likely that those patients developing the syndrome are immunologically susceptible because of their HLA status, which is similiar to that of patients with myasthenic syndrome not associated with carcinoma. It has been suggested that the latter form of myasthenic syndrome may involve a response to an antigen common to cholinergic neurons and oat-cell carcinoma. $^{724}$

Our two cases of carcinoma-associated myasthenic syndrome responded to treatment with plasma exchange and cytotoxic chemotherapy. In Case 1 alternate day steroid therapy produced minimal improvement on the day of treatment with apparent slight worsening on the steroid-free day, but this was not reflected by the electrophysiological 
findings (figs 1, 2), although slight reduction in compound muscle action potential amplitude was found in two muscles on day 3 (fig 1), a day following steroid treatment. Improvement was evident after the third plasma exchange in Case 1 , six days after beginning this treatment. This patient was also treated with cytotoxic chemotherapy, beginning on day 18 , after the fifth plasma exchange. Four treatments were given and in three months his strength had returned almost to normal, with only minimal proximal weakness in the legs. In Case 2 the response to plasma exchange was more marked in the arms than the legs and was evident four days after this treatment was commenced, after three exchanges. Three weeks after beginning plasma exchange treatment, after six exchanges, strength was normal in the arms but only slightly improved in the legs.

The response to plasma exchange in both our cases of myasthenic syndrome associated with bronchogenic carcinoma was similar to that described in 12 previously reported patients with myasthenic syndrome not associated with carcinoma ${ }^{71627}$ and two patients with myasthenic syndrome associated with carcinoma of the lung. ${ }^{78}$ This result suggests that treatment of myasthenic syndrome associated with carcinoma is worthwhile and effective, and that a similar protocol should be followed in the two groups. In our Case 1 cytotoxic chemotherapy seemed to induce a more complete and sustained remission. An earlier report ${ }^{25}$ also described improvement after successful treatment of the carcinoma but this has not been generally noted. ${ }^{28}$

The response to treatment in myasthenic syndrome can be monitored by electrophysiological tests, in particular by the size of the evoked compound muscle action potential, the effect of stimulation at rapid rates ${ }^{59}$ and the effect of short periods of voluntary activity. ${ }^{24}$ The size of the initial response increases during treatment with guanidine, ${ }^{5} \quad 4$ aminopyridine, ${ }^{11} 12 \quad 3-4$ diaminopyridine (DAP), ${ }^{13}$ plasma exchange ${ }^{7-10}$ and steroid therapy..$^{14} 153031$ Other substances also have this effect, including calcium gluconate, edrophonium and decamethonium, ${ }^{5}$ caffeine ${ }^{32}$ and choline. ${ }^{28}$

In Case 1 the amplitude of the initial compound muscle action potential in trapezius was normal following treatment but there was less marked improvement in abductor digiti minimi and extensor digitorum brevis. Following voluntary activation, the amplitude of the initial compound muscle action potential in abductor digiti minimi was normal at this time (day 45 ), but in extensor digitorum brevis the response was still small (fig 1). In both cases post-contraction facilitation of the compound mus- cle action potential was most marked in abductor digiti minimi, than in other muscles (table). The abductor digiti minimi is thus probably the most useful muscle for electrophysiological tests in myasthenic syndrome, and measurement of postcontraction compound muscle action potential is a better test than short runs of rapid repetitive nerve stimulation. Repetitive nerve stimulation for longer periods improves the diagnostic yield, but is uncomfortable. Clinical improvement with plasma exchange and immunosuppressive therapy was accompanied by slow improvement in the electrophysiological abnormality suggesting that in myasthenic syndrome not only is there interference with the release of acetyl-choline quanta ${ }^{633}$ but also that there is structural disorganisation of the presynaptic membrane active zones concerned with acetylcholine release. ${ }^{34}$

\section{References}

' Eaton LM, Lambert EH. Electromyography and electric stimulation of nerves in diseases of motor unit: observations of myasthenic syndrome associated with malignant tumors. JAMA 1957;163:1117-24.

${ }^{2}$ Lambert EH, Rooke ED, Eaton LM, Hodgson $\mathrm{CH}$. Myasthenic syndrome occasionally associated with bronchialneoplasm: neurophysiologic studies. In: Viets HR, ed. Myasthenia Gravis. Springfield, Illinois: Charles C Thomas, 1961:362-410.

${ }^{3}$ Rubenstein AE, Horowitz SH, Bender AN. Cholinergic dysautonomia and Eaton-Lambert syndrome. Neurology (Minneap) 1979;29:720-3.

${ }^{4}$ Elmquist D, Lambert EH. Detailed analysis of neuromuscular transmission in a patient with the mysasthenic syndrome sometimes associated with bronchogenic carcinoma. Mayo Clin Proc 1968; 43:689-713.

${ }^{5}$ Lambert EH. Defects of neuromuscular transmission in syndromes other than myasthenia gravis. Ann NY Acad Sci 1966;135:367-84.

- Lambert EH, Elmquist D. Quantal components of endplate potentials in the myasthenic syndrome. Ann NY Acad Sci 1971;183:183-99.

${ }^{7}$ Lang B, Newsom-Davis J, Wray D, Vincent A. Autoimmune aetiology for myasthenic (Eaton-Lambert) syndrome. Lancet 1981;2:224-6.

${ }^{8}$ Newsom-Davis J, Murray N, Wray D, et al. LambertEaton myasthenic syndrome: electrophysiological evidence for a humoral factor. Muscle Nerve 1982;5:517-20.

9 Dau PC, Denys EH. Plasmapheresis and immunosuppressive drug therapy in the Eaton-Lambert syndrome. Ann Neurol 1982;11:570-5.

${ }^{10}$ Denys EH, Dau PC, Lindstrom JM. Neuromuscular transmission before and after plasmapheresis in myasthenia gravis and the myasthenic syndrome. In: Dau PC, Plasmapheresis and the Immunobiology of Myasthenia Gravis. Boston: Houghton Miffin, 1979:24854. 
${ }^{11}$ Lundh H, Nilsson O, Rosen I. 4-aminopyridine: a new drug tested in the treatment of Eaton-Lambert syndrome. J Neurol Neurosurg Psychiatry 1977;40:1109-12.

${ }^{12}$ Murray NMF, Newsom-Davis J. Treatment with oral 4-aminopyridine in disorders of neuromuscular transmission. Neurology (NY) 1981;31:265-71.

${ }^{13}$ Lundh H, Nilsson O, Rosen I. Novel drug of choice in Eaton-Lambert syndrome. J Neurol Neurosurg Psychiatry 1983;46:684-7.

${ }^{14}$ Streib EW. Eaton-Lambert myasthenic syndrome: serial electromyographic studies during prednisone treatment. Electromyogr Clin Neurophysiol 1981;21: 107-20.

${ }^{15}$ Streib EW, Rothner AD. Eaton-Lambert myasthenic syndrome: long-term treatment of 3 patients with prednisone. Ann Neurol 1981;10:448-53.

${ }^{16}$ Newsom-Davis J. Plasmapheresis in immunological disorders of neuromuscular transmission and peripheral nerve. Apheresis Bull 1983;1:18-25.

${ }^{17}$ Newsom-Davis J, Murray N, Willcox N, Lang C, Welsh K. Lambert-Eaton myasthenic syndrome (LEMS): immunogenetic characteristics and response to immunosuppressive drug treatment. Neurology $(N Y)$ 1983; 33 (Suppl 2): 156 (abstr).

18 Portha C, Dupond JL, Monnier G, Bosset JF, Leconte des Floris R. Syndrome de Lambert et Eaton associe a un myelome multiple: reversibilite du neuromusculaire apres chimotherapie. Sem Hop Paris 1983;59:1337-9.

${ }^{19}$ Dong MM, Wasserman EJL, Giebfried J. Repetitive stimulation of the trapezius muscle: its value in myasthenic testing. Muscle Nerve 1980;3:439.

${ }^{20}$ Harvey AM, Masland RL. The electromyogram in myasthenia gravis. Johns Hopkins Med J 1941;68:81-93.

${ }^{21}$ Desmedt JE. The neuromuscular disorder in myasthenia gravis: Electrical and mechanical responses to nerve stimulation in hand muscles. In: Desmedt JE, ed. New Developments in Electromyography and Clinical Neurophysiology Vol 1. Basel: Karger, 1973:241304.

${ }^{22}$ Davis GR, Brown IT, Schwartz MS, Swash M. A dedicated microcomputer-based instrument of interval analysis of multicomponent wave forms in single fibre
EMG. Electroencephalogr Clin Neurophysiol 1983; 56: 110-3.

${ }^{23}$ Ozdemir C, Young RR. The results to be expected from electrical testing in the diagnosis of myasthenia gravis. Ann NY Acad Sci 1976;274:203-22.

${ }^{24}$ Lennon VA, Lambert EH, Whittingham S, Fairbanks V. Autoimmunity in the Lambert-Eaton myasthenic syndrome. Muscle Nerve 1982;5:21-5.

${ }^{25}$ Lambert EH, Rooke ED. Myasthenic state and lung cancer. In Brain, Lord, Norris FH, eds. The Remote Effects of Cancer on the Nervous System. New York: Grune \& Stratton, 1965:67-80.

${ }^{26}$ Trojaborg W, Frantzen E, Andersen I. Peripheral neuropathy and myopathy associated with carcinoma of the lung. Brain 1969;92:71-82.

${ }^{27}$ Kranz H, Caddy DJ, Williams AM, Gay W. Myasthenic syndrome: effect of choline, plasmapheresis and tests for circulating factor. J Neurol Neurosurg Psychiatry 1980;43:483-8.

${ }^{28}$ Henson RA, Urich H. Cancer and the Nervous System. Oxford: Blackwell Scientific, 1982:418.

${ }^{29}$ Henriksson KG, Nilsson O, Rosen I, Schiller HH. Clinical, neurophysiological and morphological findings in Eaton-Lambert syndrome. Acta Neurol Scand 1977;56: 117-40.

${ }^{30}$ Vroom FQ, Engel WK. Non-neoplastic, steroid responsive Lambert-Eaton myasthenic syndrome. Neurology 1969; 19:281 (abstr)

${ }^{31}$ Phillips LH. The use of single fiber EMG to monitor the response of Lambert-Eaton myasthenic syndrome to steroid therapy. Electroencephalogr Clin Neurophysiol 1983;55: 43 (abstr).

32 Takamori M. Caffeine, calcium and Eaton-Lambert syndrome. Arch Neurol 1972;27:285-91.

${ }^{33}$ Lindstrom JM, Lambert EH. Content of acetylcholine receptor and antibodies bound to receptor in myasthenia gravis, experimental autoimmune myasthenia gravis and Eaton-Lambert syndrome. Neurology (Minneap) 1978;28:130-8.

${ }^{34}$ Fukunaga H, Eugel AG, Osame M, Lambert EH. Paucity and disorganisation of presynaptic membrane active zones in the Lambert-Eaton myasthenic syndrome. Muscle Nerve 1982;5:686-97. 